\title{
Surface-state spin textures in strained bulk HgTe: Strain-induced topological phase transitions
}

\author{
Frank Kirtschig, ${ }^{1}$ Jeroen van den Brink, ${ }^{1,2,3}$ and Carmine Ortix ${ }^{1,4}$ \\ ${ }^{1}$ Institute for Theoretical Solid State Physics, IFW Dresden, PF 270116, 01171 Dresden, Germany \\ ${ }^{2}$ Department of Physics, Dresden University of Technology, 01062 Dresden, Germany \\ ${ }^{3}$ Department of Physics, Harvard University, Cambridge, Massachusetts 02138, USA \\ ${ }^{4}$ Institute for Theoretical Physics, Center for Extreme Matter and Emergent Phenomena, Utrecht University, Leuvenlaan 4, \\ 3584 CE Utrecht, Netherlands \\ (Received 1 May 2016; published 27 December 2016)
}

\begin{abstract}
The opening of a band gap due to compressive uniaxial strain renders bulk HgTe a strong three-dimensional topological insulator with protected gapless surface states at any surface. By employing a six-band $\mathbf{k} \cdot \mathbf{p}$ model, we determine the spin textures of the topological surface states of bulk HgTe uniaxially strained along the (100) direction. We show that at the (010) and (001) surfaces, an increase in the strain magnitude triggers a topological phase transition where the winding number of the surface-state spin texture is flipped while the four topological invariants characterizing the bulk band structure of the material are unchanged.
\end{abstract}

DOI: 10.1103/PhysRevB.94.235437

\section{INTRODUCTION}

Topological insulators (TIs) are new quantum states of matter whose theoretical prediction and experimental verification has had a tremendous impact in the field of fundamental condensed matter physics [1-17], and for potential applications in spintronics and quantum computation [18]. Time-reversal (TR) invariant TIs are insulating in the bulk but they do possess gapless surface states topologically protected by TR symmetry [1,3]. These metallic surface states are spin-momentum locked: surface electrons with opposite spins counterpropagate at the sample boundaries. For three-dimensional (3D) TIs, $\mathrm{Bi}_{2} \mathrm{Se}_{3}$ [10], $\mathrm{Bi}_{14} \mathrm{Rh}_{3} \mathrm{I}_{9}$ [15], and $\beta-\mathrm{HgS}[19,20]$ to name but a few, the existence of these topological surface states (TSS) can be directly inferred from the four $\mathbb{Z}_{2}$ indices characterizing the bulk band structure of a 3D TR invariant insulator [7-9]. However, both the strong $v_{0}$ index and the three weak $\left\{v_{1}, v_{2}, v_{3}\right\}$ indices make no assertion on the nature of the spin textures of the surface states which, realizing a vortex structure in momentum space, can be characterized topologically by the winding number (the topological charge of the vortex) of the planar unit spin $\left(n_{i}, n_{j}\right)=\left(S_{i}, S_{j}\right) / \sqrt{S_{i}^{2}+S_{j}^{2}}$. It is defined by

$$
w=\oint_{\mathbb{C}} \frac{d \mathbf{k}}{2 \pi} \cdot\left[n_{i} \nabla_{\mathbf{k}} n_{j}-n_{j} \nabla_{\mathbf{k}} n_{i}\right]
$$

where $\mathbb{C}$ is a closed loop in momentum space encircling the essential degeneracy point of the topological surface state, guaranteed by TR invariance. Generally speaking, the two winding numbers $w= \pm 1$ (cf. Fig. 1) are equally compatible for linear Dirac cones, and the specific value is independent of the $\mathbb{Z}_{2}$ topological indices of the bulk band structure. In many strong 3D TIs with a single Dirac cone on the surface, however, additional point group symmetries at the surfaces pin the spin texture winding number to $w=1$. This occurs for instance at the high $\mathcal{C}_{3 v}$ [21,22] symmetry surfaces of materials with a rhombohedral crystal structure such as $\mathrm{Bi}_{2} \mathrm{Se}_{3}$. For surfaces where the symmetry is lowered, a similar assertion cannot be made.
In this paper, we show that at surfaces with point group symmetry lower than $\mathcal{C}_{2 v}$, the surface-state spin textures of compressively strained bulk $\mathrm{HgTe}-\mathrm{a}$ strong 3D TI whose nontrivial topological properties have been experimentally verified by quantum Hall measurements [23] - have a topological charge that can be flipped from $w=1$ to $w=-1$ by continuously increasing the strain magnitude. By employing a six-band $\mathbf{k} \cdot \mathbf{p}$ Kane model, we demonstrate that for a uniaxial strain along the (100) direction, the orbital character of the topological surface states at the (010) and (001) surfaces depends sensitively upon the strain magnitude. This ultimately leads to a change in the nature of the surface-state spin textures at a critical strain magnitude.

The paper is organized as follows: in Sec. II we introduce the six-band $\mathbf{k} \cdot \mathbf{p}$ model for uniaxially strained $\mathrm{HgTe}$ and verify the occurrence in semi-infinite slab geometries of a surface Kramer's doublet at the surface Brillouin zone (BZ) center; in Sec. III we define mirror Chern numbers for the long-wavelength $\mathbf{k} \cdot \mathbf{p}$ model and define ensuing pseudospin textures for the surface Dirac cones, directly linked to the spin textures (see Sec. IV). In Sec. V we introduce an inversion symmetry breaking perturbation, thanks to which the $\mathbf{k} \cdot \mathbf{p}$ model correctly captures the $T_{d}$ point group symmetry of the zinc-blende lattice, and verify that the strain-induced topological phase transition for the spin textures is still present. Finally we draw our conclusions in Sec. VI.

\section{TOPOLOGICAL SURFACE-STATE DIRAC POINTS}

Pristine HgTe is a zero gap semiconductor with the Fermi energy in the middle of the fourfold degenerate light-hole $(\mathrm{LH})$ and heavy-hole $(\mathrm{HH}) \Gamma_{8}$ states at the BZ center [7,24]. The topological nature of the electronic states in this material cannot be inferred from these $p_{3 / 2}$ atomic levels [7] but rather follows from the inverted band ordering at the zone center of the LH $\Gamma_{8}$ band, which is particlelike, and the $\Gamma_{6} s$ band, which is holelike. In normal semiconductors, such as $\mathrm{CdTe}$, the $\Gamma_{6}$ band forms the conduction band while the $\mathrm{LH}$ $\Gamma_{8}$ band represents one of the valence bands. This inverted band ordering, which is an immediate consequence of the strong spin-orbit coupling of $\mathrm{Hg}$, establishes this material 

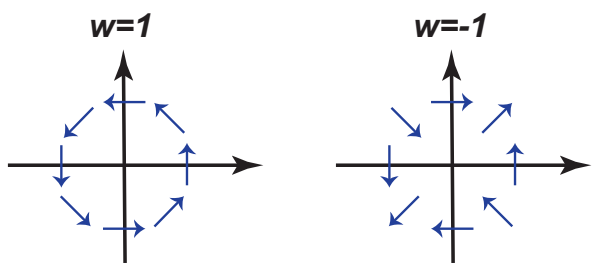

FIG. 1. Sketch of possible spin textures of Dirac-like surface states in strong 3D TI. The left panel corresponds to a right-handed helical structures with winding number $w=1$. In the right panel $w=-1$.

to be topologically nontrivial since two bands of opposite parities [25] have level crossed with respect to the normal band ordering. By externally applying a compressive uniaxial strain, the fourfold degeneracy of the $\Gamma_{8}$ states at the zone center is lifted and thus a gap at the Fermi energy opens up [7,23]. This immediately establishes compressively strained $\mathrm{HgTe}$ as a strong 3D TI.

The bulk-boundary correspondence $[16,17]$ then guarantees the existence of TSS with a conical dispersion at any surface, and the surface Kramer's doublet— the Dirac point—sitting at the surface BZ center. To verify this, we rely on an effective low-energy theory based upon a $\mathbf{k} \cdot \mathbf{p}$ expansion of the lowest energy bands around the $\Gamma$ point of the BZ. This approach has successfully described the quantum spin Hall effect in $\mathrm{HgTe} / \mathrm{CdTe}$ quantum wells $[4,6]$. We thus employ the six-band Kane model for the $\Gamma_{6,8}$ bands [26], where the influence of a compressive uniaxial strain, which, without loss of generality, we assume along the $\hat{x}$ direction, is taken into account via the Bir-Pikus Hamiltonian (see the Supplemental Material [27]). The Luttinger and $\mathbf{k} \cdot \mathbf{p}$ parameters are based on the $T=0$ band structure of pristine HgTe [28]. We can establish the presence and the electronic characteristic of the surface Kramer's doublet at the (100), (010), and (001) surfaces by solving the $\mathbf{k} \cdot \mathbf{p}$ model at the surface $\mathrm{BZ}$ center in the half-infinite space $x \lessgtr 0, y \lessgtr 0$, and $z \lessgtr 0$, respectively, using the general method outlined in Ref. [29].

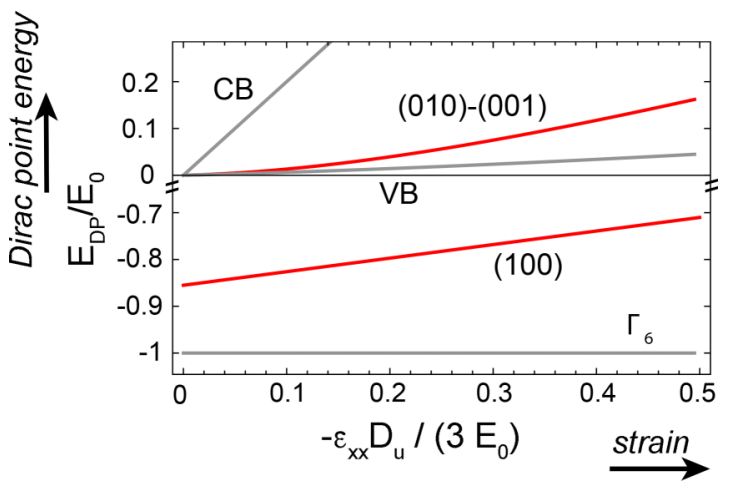

FIG. 2. Behavior of the surface Dirac point in compressively strained $\mathrm{HgTe}$ as a function of the strain magnitude $\epsilon_{x x}$ at the (100), (010), and (001) surfaces (red lines). We also show the conduction and valence band edges as well as the $\Gamma_{6}$ band edge (gray lines). At the (100) surface, the surface Dirac point is buried within the $\mathrm{HH}$ valence band but resides in the band gap of the LH- $\Gamma_{6}$ TI bulk.

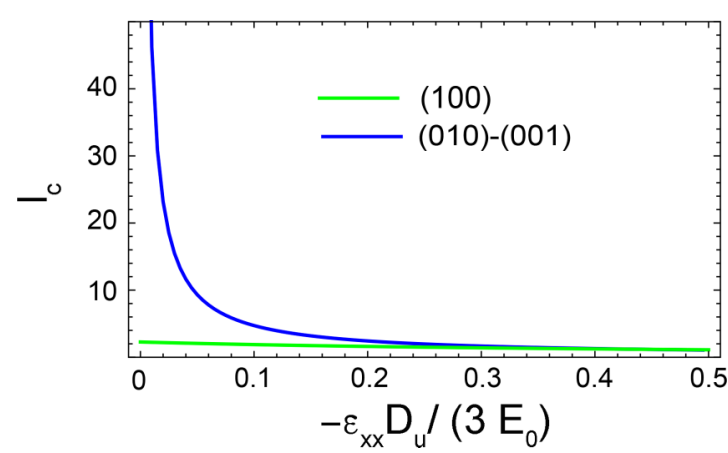

FIG. 3. Behavior of the topological surface-state penetration depth in compressively strained $\mathrm{HgTe}$ as a function of the strain magnitude $\epsilon_{x x}$ at the (100), (010), and (001) surfaces.

Figure 2 shows the behavior of the surface Dirac point energy $E_{D P}$ as a function of the uniaxial strain magnitude $\epsilon_{x x}$ renormalized by the factor $D_{u} /\left(3 E_{0}\right)$ where $E_{0}$ is the distance of the $\Gamma_{6}$ band edge from the direct BZ center midgap while $D_{u}$ is the deformation potential of $\mathrm{HgTe}$. At the (100) surface, the surface Kramer's doublet is buried within the $\mathrm{HH}$ valence band, while on the two other surfaces it resides in the indirect bulk gap of the system. This termination dependence is also reflected in the behavior of the penetration depth of the surface states (cf. Fig. 3). Specifically, the surface states at the (010) and (001) surfaces are characterized by a diverging decay length in the $\epsilon_{x x} \rightarrow 0$ limit, which implies that at these planes the TSS penetrate more deeply into the bulk as compared to the (100) TSS.

These different electronic characteristics can be attributed to the different nature of the Dirac wave function of the surface BZ center. At the (100) plane, indeed, the surface-state Dirac wave function is all made of $\mathrm{LH}$ and $\Gamma_{6}$ states. A uniaxial strain along the $\hat{x}$ direction preserves the axial rotation symmetry in the plane, and thus at the surface $\Gamma$ point with momentum $k_{y} \equiv k_{z} \equiv 0$ the total angular momentum $J_{x}$ is a good quantum number [4,29]. This, in turn, implies the absence of any mixing between the $\left|J=3 / 2 ; J_{x}= \pm 3 / 2\right\rangle \mathrm{HH}$ states and the $J_{x}= \pm 1 / 2 \mathrm{LH}$ and $\Gamma_{6}$ states. Henceforth, the HH bands play the role of inserted "parasitic" bands [29] on top of the LH- $\Gamma_{6}$ TI bulk, in the band gap of which the surface Dirac point resides (cf. Fig. 2). This does not hold true at the (010) and (001) planes where the uniaxial strain along the $\hat{x}$ direction breaks the in-plane rotation symmetry, thereby leading to an effective hybridization between the $J_{y, z}= \pm 1 / 2$ states with $J_{y, z}= \pm 3 / 2 \mathrm{HH}$ states. The surface Dirac wave function becoming a superposition of $\Gamma_{6,8}$ localized states is then pushed out of the HH bulk bandwidth [30] and remerges in the full band gap of the system, in agreement with the features encountered in the Fano model [31].

\section{MIRROR CHERN NUMBERS AND PSEUDOSPIN TEXTURES}

To proceed further, we now introduce the notion of mirror Chern numbers (MCN). The MCN are topological invariants, which are protected by mirror symmetries. In the absence of strain and neglecting the bulk inversion asymmetry of the zinc-blende crystal structure, the point group at the $\Gamma$ 
point of $\mathrm{HgTe}$ is the full cubic group $O_{h}$. Correspondingly the Kane model Hamiltonian is invariant under nine mirror symmetry operations. The presence of an uniaxial strain along the (100) direction reduces the number of these mirror symmetry operations but preserves the ones with respect to the (100), (010), and (001) planes. Since the Kane model Hamiltonian commutes with the corresponding mirror symmetry operations at the three planes $k_{x, y, z} \equiv 0$, all eigenstates can be classified according to their $\pm i$ mirror parity. This allows one to define two time-reversal related Chern numbers $\mathcal{C}_{ \pm i}$ whose sum vanishes but with a difference $n_{\mathcal{M}}=\left(\mathcal{C}_{i}-\mathcal{C}_{-i}\right) / 2$, which is an integer $\mathbb{Z}$ topological invariant and defines the MCN.

We have computed the MCNs of the full six-band Kane model Hamiltonian using its decomposition at the mirror planes in terms of the nine Gell-Mann matrices, and subsequently employed the elegant formulation of Ref. [32] to derive the Chern numbers for the corresponding continuum models. This allows us to avoid an effective two-band modeling which can only be introduced ad hoc. We find a MCN $n_{\mathcal{M}} \equiv-1$ at the $k_{y, z} \equiv 0$ planes, whereas $n_{\mathcal{M}} \equiv 1$ at the $k_{x} \equiv 0$ plane. And indeed, under proper coordinate transformations, the continuum $\mathbf{k} \cdot \mathbf{p}$ Hamiltonians at the $k_{y, z} \equiv 0$ planes cannot be adiabatically transformed into the $k_{x} \equiv 0$ one without closing the bulk band gap. The three MCNs indicated above allow us to immediately derive a pseudospin texture for the TSS at the (100), (010), and (001) planes, as explained below. At the (100) surface, the projection of the two (010), (001) planes define two lines where the TSS can be classified according to their mirror eigenvalues. We can thus define a pseudospin vector $\sigma$ with components related to the mirror operators $\mathcal{M}_{y, z}$ by $\sigma_{y, z}=-i \mathcal{M}_{y, z}$. The dispersion of the TSS can be then written in terms of this pseudospin as

$$
H_{e f f}^{(100)}=v_{F}^{z} k_{y} \sigma_{z}-v_{F}^{y} k_{z} \sigma_{y},
$$

where the sign of the two Fermi velocities $v_{F}^{z, x}$ is uniquely determined by the MCNs of the system via the bulk-edge correspondence for the $k_{y, z} \equiv 0$ planes. Specifically we have $\operatorname{sgn}\left(v_{F}^{z, y}\right)=n_{\mathcal{M}}^{k_{z, y}=0}$ which yields the pseudospin texture shown in the top panel of Fig. 4(a). It exhibits a helical structure with a left-handed helicity for the surface-state conduction band, and a right-handed one for the valence band, in perfect agreement with density functional theory studies [33,34]. At the opposite (100) surface the sign of both Fermi velocities are flipped, which changes the helicity of the pseudospin texture but still preserves the pseudospin texture winding number $w=\operatorname{sgn}\left(v_{F}^{z} \times v_{F}^{y}\right)=1$. A similar analysis at the (010) and (001) surface terminations yields the pseudospin textures shown in Fig. 4(a). At these surfaces the different values of the two MCN yield a pseudospin texture with an opposite winding number $w=-1$.

Figure 5 shows the behavior of the magnitude of the TSS Fermi velocities $\left|v_{F}\right|$ as a function of the strain magnitude $\epsilon_{x x}$. We find that for the TSS at the (100) plane, the two Fermi velocities $v_{F}^{y, z}$ have equal magnitudes. Therefore, the TSS display a global $\mathcal{U}(1)$ rotational symmetry similar to the case of, for instance, $\mathrm{Bi}_{2} \mathrm{Se}_{3}$ [21]. As a two-dimensional $\mathbf{k} \cdot \mathbf{p}$ for the pseudospin one-half surface Kramer doublet explicitly shows [29], this is an immediate consequence of the fourfold rotational symmetry along the $\hat{x}$ axis of the Kane model

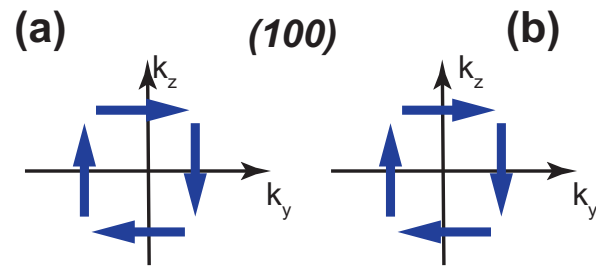

(010)

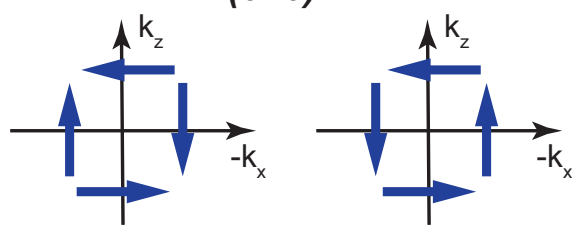

(001)

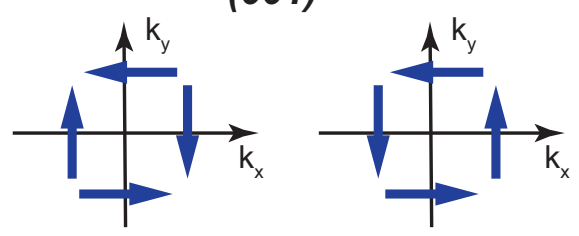

FIG. 4. (a) Pseudospin textures for the TSS of HgTe in the presence of a uniaxial strain along the (100) direction at the (100) (top panels), (010) (middle panels), and (001) (bottom panels) crystal planes. (b) Physical spin textures for strain smaller than the critical one $-\epsilon_{x x}<-\epsilon_{c}$. For larger strain the physical spin textures correspond to the pseudospin ones.

Hamiltonian. And indeed, at the (010) and (001) planes where the uniaxial strain along the (001) direction lowers the surface point group symmetry, we find the Fermi velocities of the TSS to differ, with an anisotropy that is enhanced by increasing the strain magnitude.

\section{SPIN TEXTURES}

The pseudospin textures of the TSS have a close relation to the physical spin textures. This can be found introducing the projector operator $\mathcal{P}_{x, y, z}=\left(\mathbb{1}-i \mathcal{M}_{x, y, z}\right) / 2$ onto the subspaces of the Kane model Hamiltonian with mirror parity $+i$, and considering their effect on the physical spin operators

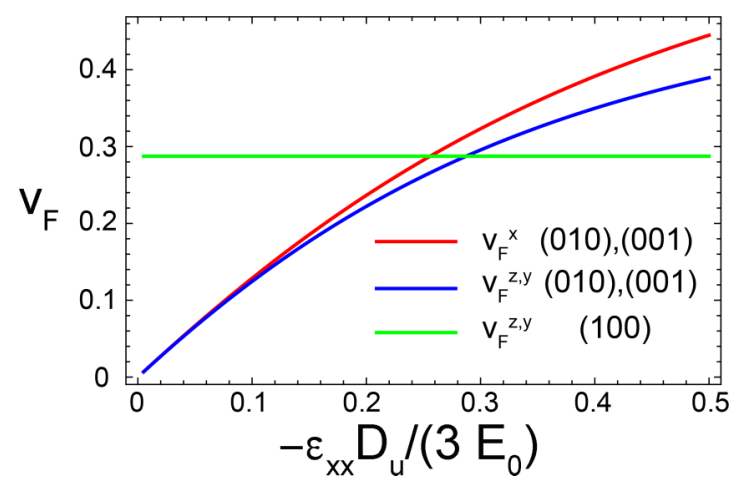

FIG. 5. Behavior of the magnitudes of the Fermi velocities $\left|v_{F}\right|$ in the TSS of strained $\mathrm{HgTe}$ as a function of the strain magnitude $\epsilon_{x x}$. The TSS at the (010) and (001) planes exhibit an anisotropic behavior since the uniaxial strain along the $\hat{x}$ direction lowers the point group symmetry. 
$\mathcal{S}_{x, y, z}$ at the planes invariant under the mirror symmetry operations. It can be shown that $\mathcal{P}_{i} \mathcal{S}_{j} \mathcal{P}_{i} \equiv 0$ for $i \neq j$, which simply states that at the surface lines invariant under the mirror symmetries, the physical spin can be either parallel or antiparallel to the pseudospin. For $\mathrm{SmB}_{6}$, the physical spin was found to be always parallel to the pseudospin, and thus the knowledge of the MCNs provides a robust classification of the topological surface-state spin textures [35]. This, however, does not hold true for $\mathrm{HgTe}$. The projected spin operator of the $\Gamma_{6,8}$ bands has both positive and negative eigenvalues, and thus the relation between the pseudospin and the physical spin depends on the orbital composition of the surface states. By evaluating the projected spin operator eigenvalues of the TSS along the aforementioned symmetry invariant lines, we find that the physical spin is always parallel to the pseudospin except at the $k_{z} \equiv 0$ and $k_{y} \equiv 0$ lines for the (010) and (001) surface terminations, respectively. At these lines indeed, the physical spin is antiparallel to the pseudospin below a critical strain magnitude $\left|\epsilon_{c}\right|$, and parallel above it. This implies that contrary to the TSS pseudospin textures at the (010) and (001) surface terminations, which are characterized by a $w=-1$ winding number independent of the strain magnitude, the physical spin textures exhibit a right-handed $w=1$ helical structure for small strain [cf. Fig. 5(b)]. The $w=-1$ spin textures equal to the pseudospin textures are then restored for larger strain values.

To gain more insight into this spin texture topological phase transition, we have computed the orbital resolved projected spin eigenvalue of the TSS along the mirror invariant lines $k_{y, z} \equiv 0$ for the (001) and (010) surface terminations, respectively, by varying the strain magnitude (cf. Fig. 6). The TSS with positive pseudospin is an admixture of $\left|J, J_{y, z}\right\rangle=|1 / 2,1 / 2\rangle \Gamma_{6},|3 / 2,3 / 2\rangle \mathrm{HH}$ and $|3 / 2,-1 / 2\rangle$ LH states. For small strain, the TSS has a predominant LH orbital character which implies that the physical spin is antiparallel to the pseudospin. By continuously increasing the strain magnitude, the TSS starts to acquire a sizable $\Gamma_{6}$ and $\mathrm{HH}$ character which ultimately reverses the spin direction to be parallel to the pseudospin.

\section{BULK INVERSION ASYMMETRY EFFECTS}

Next, we take into account the bulk inversion asymmetry (BIA) of the zinc-blende crystal structure, with point group

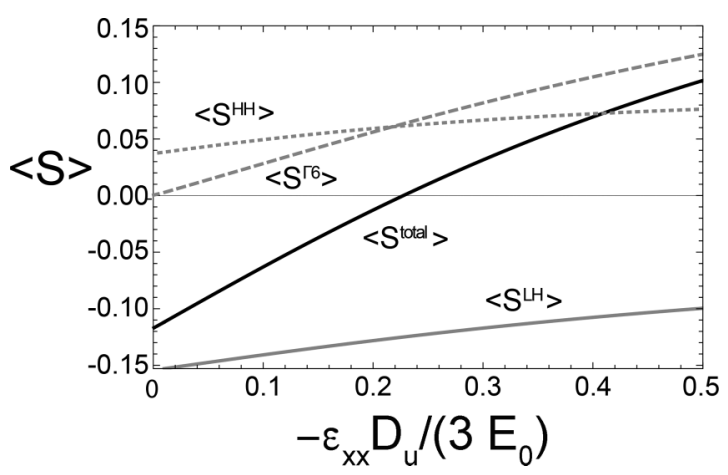

FIG. 6. Orbital resolved (gray lines) and total spin (black line) of the TSS with positive pseudospin for the (010) [(001)] plane at the $k_{z} \equiv 0\left[k_{y} \equiv 0\right]$ line. For small strain, the physical spin is antiparallel to the pseudospin, while for large strain they are parallel. symmetry $T_{d}[26,36]$. From a $\mathbf{k} \cdot \mathbf{p}$ perspective, this point group symmetry reduction yields additional linear in $\mathbf{k}$ terms in the $\Gamma_{8}$ block Hamiltonian, which originate from bilinear couplings consisting of $\mathbf{k} \cdot \mathbf{p}$ and spin-orbit interaction with the uppermost $d$ core levels [37,38]. They read

$$
\mathcal{H}_{\mathrm{BIA}}^{8,8}=c\left[\left\{J_{x}, J_{y}^{2}-J_{z}^{2}\right\} k_{x}+c . p \cdot\right] / \sqrt{3},
$$

where $J_{x, y, z}$ are the $J=3 / 2$ angular momentum matrices, c.p. denotes the cyclic permutations of the preceding term, while $\left\{J_{i}, J_{j}\right\}$ denotes the anticommutator of $J_{i}$, and $J_{j}$. Finally, $c$ is an elementary parameter of the Kane model Hamiltonian [26].

The presence of the BIA terms destroys the mirror symmetry operations of the $\mathbf{k} \cdot \mathbf{p}$ Hamiltonian at the (100), (010), and (001) plane, and consequently the spin textures of the TSS cannot be directly inferred from the mirror Chern number discussed above. However, the spin textures can be topologically characterized by their winding number since they yet realize a planar vortex structure in momentum space as we show below.

In presence of a uniaxial strain along the (100) direction, the point group symmetry $T_{d}$ is reduced to the tetragonal symmetry $D_{2 d}$ [39]. Therefore, at the (010) and (001) surface the system still retains a twofold rotation symmetry along the $y$ and $z$, respectively. By choosing as a natural basis for the surface Kramers doublet sitting at the surface BZ center the total
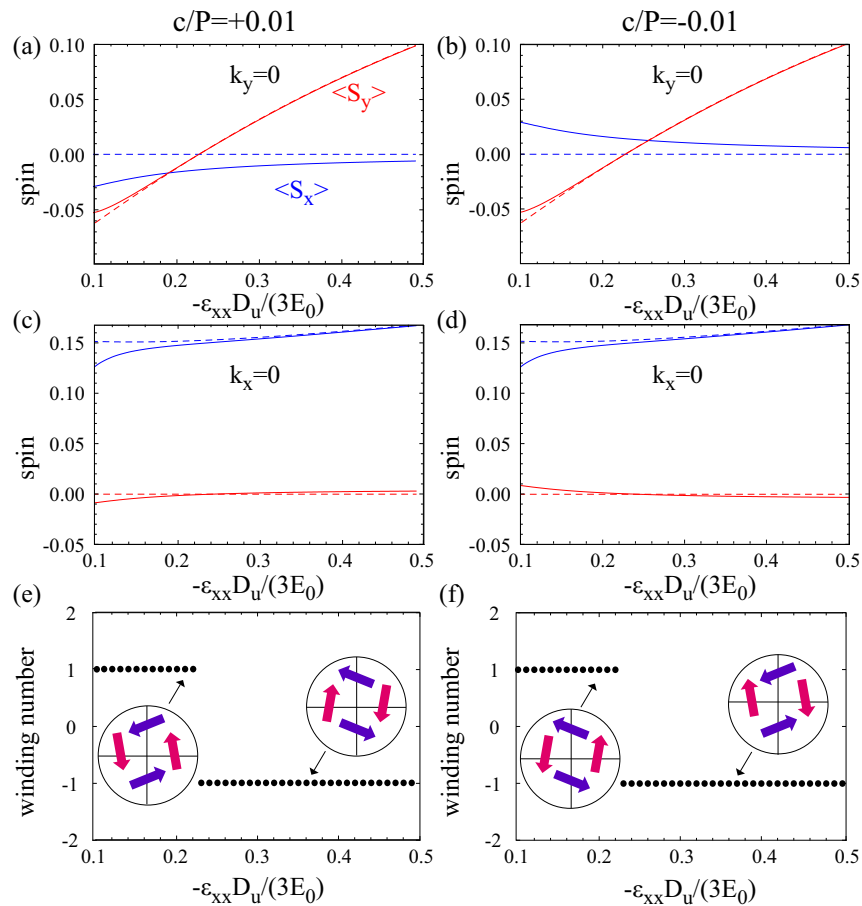

FIG. 7. Behavior of the spin components of the TSS at the (001) surface at the $k_{y}=0$ line for $k_{x}<0$ (a),(b) and at the $k_{x}=0$ line for $k_{y}<0$ (c),(d) as a function of the strain magnitude $\epsilon_{x x}$ including BIA terms in the Kane model. The dashed lines are the results in the absence of BIA terms where the spin textures are determined by mirror Chern numbers. We have chosen two representative values of the elementary parameter $c$. Panels (e) and (f) sketch the corresponding spin textures and show the calculated winding number as a function of the strain magnitude. 


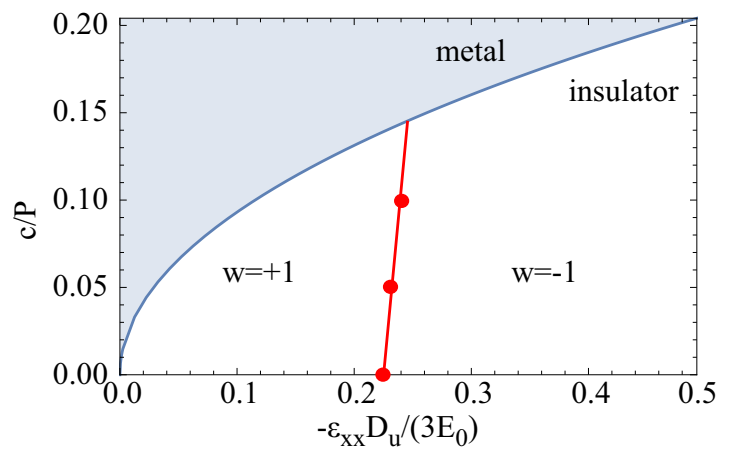

FIG. 8. Phase diagram for the winding number of the spin texture in the $\epsilon_{x x}-c$ plane. The colored area corresponds to regions where the Kane model does not predict the opening of an indirect bulk band gap. The red line separates the two regions of opposite winding numbers.

angular momentum $J_{z}= \pm 1 / 2$ [21], we have that the twofold rotational symmetry can be represented as $\mathcal{C}_{2}=-i \sigma_{z}$. On the contrary the antiunitary time-reversal symmetry operator can be represented as usual by $\Theta=i \sigma_{y} \mathcal{K}$, where $\mathcal{K}$ is the complex conjugate. The form of the $\mathbf{k} \cdot \mathbf{p}$ surface Hamiltonian is then constrained by the time reversal and rotational symmetry. Indeed under $\mathcal{C}_{2}$, spin and momentum transform according to $k_{x, y} \rightarrow-k_{x, y}, \sigma_{x, y} \rightarrow-\sigma_{x, y}$, and $\sigma_{z} \rightarrow \sigma_{z}$. With the timereversal symmetry constraint being $\mathcal{H}(\mathbf{k})=\sigma_{y} \mathcal{H}^{\star}(-\mathbf{k}) \sigma_{y}$, the linear in $\mathbf{k}$ surface Hamiltonian reads

$$
\mathcal{H}_{e f f}(\mathbf{k})=v_{F}^{y} k_{x}\left(\sigma_{y}-\alpha \sigma_{x}\right)-v_{F}^{x} k_{y}\left(\sigma_{x}-\beta \sigma_{y}\right),
$$

which, although allowing for a distortion of the textures presented in Fig. 4, still yields a completely planar structure.

To verify that the topological charge of the spin textures and the ensuing strain-induced topological phase transition are not affected by the inversion symmetry breaking perturbation, we have solved the Kane model Hamiltonian with BIA terms in the half-infinite space $z<0$. Figure 7 shows the spin components of the TSS at the $k_{x, y} \equiv 0$ lines for $k_{y, x}<0$ as a function of the strain magnitude at two representative values of the BIA parameter $c$. The TSS spin ceases to be orthogonal to these lines acquiring a finite component parallel to them. The spin textures then gets tilted but still conserves the winding number as an explicit calculation shows. Moreover, as found earlier (cf. bottom panels in Fig. 4), the $\hat{y}$ spin component at the $k_{y}=0$ line switches sign at a critical strain, thereby confirming the presence of the strain-induced topological phase transition where the winding number of the spin textures switches from $w=1$ to $w=-1$. Figure 8 shows a phase diagram for the spin textures winding number obtained by varying the strength of the BIA term. The critical strain at which the winding number of the spin textures is flipped depends very weakly on the $\mathbf{k} \cdot \mathbf{p}$ elementary parameter $c$, demonstrating that the occurrence of the topological phase transition is independent of BIA effects.

\section{CONCLUSIONS}

We have classified the topological surface-state spin texture of bulk uniaxially strained $\mathrm{HgTe}$, and have shown that assuming a strain along the $\hat{x}$ direction, the spin texture at the (100) plane exhibits a conventional left-handed helical structure, while considering (010) and (001) surface terminations the nature of the spin texture strongly depends on the orbital character of the topological surface states. The topological charge of the spin texture vortex structure can be indeed flipped by increasing the strain magnitude. This phenomenon is entirely due to the strain dependence of the orbital character of the TSS and occurs without any bulk band gap closingreopening point making such a topological phase transition very different from the ones proposed in, for instance, $\mathrm{SmB}_{6}$ [35] and $\mathrm{HgTe}_{x} \mathrm{~S}_{1-x}$ [34]. Finally, our results can be potentially tested using spin-resolved ARPES in strained HgTe.

\section{ACKNOWLEDGMENTS}

We acknowledge the financial support of the Future and Emerging Technologies (FET) programme within the Seventh Framework Programme for Research of the European Commission under FET-Open Grant No. 618083 (CNTQC). This work has been supported by the Deutsche Forschungsgemeinschaft under Grants No. OR 404/1-1 and No. SFB 1143. J.v.d.B. acknowledges support from the Harvard-MIT Center for Ultracold Atoms.
[1] C. L. Kane and E. J. Mele, Phys. Rev. Lett. 95, 146802 (2005).

[2] C. L. Kane and E. J. Mele, Phys. Rev. Lett. 95, 226801 (2005).

[3] L. Fu and C. L. Kane, Phys. Rev. B 74, 195312 (2006).

[4] B. A. Bernevig, T. L. Hughes, and S.-C. Zhang, Science 314, 1757 (2006).

[5] C. Wu, B. A. Bernevig, and S.-C. Zhang, Phys. Rev. Lett. 96, 106401 (2006).

[6] M. König, S. Wiedmann, C. Brüne, A. Roth, H. Buhmann, L. W. Molenkamp, X.-L. Qi, and S.-C. Zhang, Science 318, 766 (2007).

[7] L. Fu and C. L. Kane, Phys. Rev. B 76, 045302 (2007).

[8] L. Fu, C. L. Kane, and E. J. Mele, Phys. Rev. Lett. 98, 106803 (2007).

[9] J. E. Moore and L. Balents, Phys. Rev. B 75, 121306 (2007).
[10] H. Zhang, C.-X. Liu, X.-L. Qi, X. Dai, Z. Fang, and S.-C. Zhang, Nat. Phys. 5, 438 (2009).

[11] D. Hsieh, D. Qian, L. Wray, Y. Xia, Y. S. Hor, R. J. Cava, and M. Z. Hasan, Nature (London) 452, 970 (2008).

[12] Y. Xia, D. Qian, D. Hsieh, L. Wray, A. Pal, H. Lin, A. Bansil, D. Grauer, Y. S. Hor, R. J. Cava, and M. Z. Hasan, Nat. Phys. 5, 398 (2009).

[13] Y. L. Chen, J. G. Analytis, J.-H. Chu, Z. K. Liu, S.-K. Mo, X. L. Qi, H. J. Zhang, D. H. Lu, X. Dai, Z. Fang, S. C. Zhang, I. R. Fisher, Z. Hussain, and Z.-X. Shen, Science 325, 178 (2009).

[14] D. Hsieh, Y. Xia, L. Wray, D. Qian, A. Pal, J. H. Dil, J. Osterwalder, F. Meier, G. Bihlmayer, C. L. Kane, Y. S. Hor, R. J. Cava, and M. Z. Hasan, Science 323, 919 (2009). 
[15] B. Rasche, A. Isaeva, M. Ruck, S. Borisenko, V. Zabolotnyy, B. Büchner, K. Koepernik, C. Ortix, M. Richter, and J. van den Brink, Nat. Mater. 12, 422 (2013).

[16] M. Z. Hasan and C. L. Kane, Rev. Mod. Phys. 82, 3045 (2010).

[17] X.-L. Qi and S.-C. Zhang, Rev. Mod. Phys. 83, 1057 (2011).

[18] A. R. Akhmerov, J. Nilsson, and C. W. J. Beenakker, Phys. Rev. Lett. 102, 216404 (2009)

[19] F. Virot, R. Hayn, M. Richter, and J. van den Brink, Phys. Rev. Lett. 106, 236806 (2011).

[20] F. Virot, R. Hayn, M. Richter, and J. van den Brink, Phys. Rev. Lett. 111, 146803 (2013).

[21] L. Fu, Phys. Rev. Lett. 103, 266801 (2009).

[22] C. X. Liu, X. L. Qi, H. J. Zhang, X. Dai, Z. Fang, and S. C. Zhang, Phys. Rev. B 82, 045122 (2010).

[23] C. Brüne, C. X. Liu, E. G. Novik, E. M. Hankiewicz, H. Buhmann, Y. L. Chen, X. L. Qi, Z. X. Shen, S. C. Zhang, and L. W. Molenkamp, Phys. Rev. Lett. 106, 126803 (2011).

[24] R.-L. Chu, W.-Y. Shan, J. Lu, and S.-Q. Shen, Phys. Rev. B 83, 075110 (2011).

[25] The effect of the bulk inversion asymmetry of the zinc-blende crystal structure in mercury chalcogenides can be considered as a small perturbation [7], which does not hinder the topological nature of the level crossing.

[26] R. Winkler, Spin-Orbit Coupling Effects in Two-Dimensional Electron and Hole Systems (Springer, Berlin, 2003).
[27] See Supplemental Material at http://link.aps.org/supplemental/ 10.1103/PhysRevB.94.235437 for detailed information about the Kane model Hamiltonian, and the computation of mirror Chern numbers in three-band systems.

[28] E. G. Novik, A. Pfeuffer-Jeschke, T. Jungwirth, V. Latussek, C. R. Becker, G. Landwehr, H. Buhmann, and L. W. Molenkamp, Phys. Rev. B 72, 035321 (2005).

[29] C. Ortix, J. W. F. Venderbos, R. Hayn, and J. van den Brink, Phys. Rev. B 89, 121408(R) (2014).

[30] D. L. Bergman and G. Refael, Phys. Rev. B 82, 195417 (2010).

[31] U. Fano, Phys. Rev. 124, 1866 (1961).

[32] R. Barnett, G. R. Boyd, and V. Galitski, Phys. Rev. Lett. 109, 235308 (2012)

[33] Q.-Z. Wang, S.-C. Wu, C. Felser, B. Yan, and C.-X. Liu, Phys. Rev. B 91, 165435 (2015).

[34] T. Rauch, S. Achilles, J. Henk, and I. Mertig, Phys. Rev. Lett. 114, 236805 (2015).

[35] M. Legner, A. Rüegg, and M. Sigrist, Phys. Rev. Lett. 115, 156405 (2015).

[36] X. Dai, T. L. Hughes, X.-L. Qi, Z. Fang, and S.-C. Zhang, Phys. Rev. B 77, 125319 (2008).

[37] G. Dresselhaus, Phys. Rev. 100, 580 (1955).

[38] M. Cardona, N. E. Christensen, and G. Fasol, Phys. Rev. Lett. 56, 2831 (1986).

[39] J. Ruan, S.-K. Jian, H. Yao, H. Zhang, S.-C. Zhang, and D. Xing, Nat. Commun. 7, 11136 (2016). 